\title{
The importance of the SIBLING family of proteins on skeletal mineralisation and bone remodelling
}

\section{Katherine A Staines, Vicky E MacRae and Colin Farquharson}

The Roslin Institute and Royal (Dick) School of Veterinary Studies, The University of Edinburgh, Easter Bush, Edinburgh, Midlothian EH25 9RG, UK

Correspondence should be addressed to $K$ A Staines

Email

kstaines@rvc.ac.uk
The authors and journal apologize for an error in the above paper, which appeared in volume 214 part 3, pages 241-255. The sentence on page 246 'The Akp-2-deficient mouse displays a similar decreased $\mathrm{PP}_{\mathrm{i}}$ and OPN with an associated hypomineralisation' should read 'In contrast, the $A k p 2$-deficient mouse displays increased $\mathrm{PP}_{\mathrm{i}}$ and $\mathrm{OPN}$ with an associated hypomineralisation'. 